\title{
Editorial: Pediatric Research: Substance and Process
}

\author{
JOSEPH W. ST. GEME, JR. \\ Department of Pediatrics, UCLA School of Medicine, Marbor-UCLA Medical Center. Torrance, California. USA
}

Several of us have become involved during the past year in an informal effort to augment pediatric research and the congressional activity necessary to supply the dollars for fundamental and clinical investigation concerning mothers, infants, children, and adolescents. I, for one, was surprised by the rather cheerful remarks of Dr. Lowe at the APA-APS-SPR Annual Meeting in San Antonio last spring. Our pediatric research societies, the Association of Medical School Pediatric Department Chairmen, and the American Academy of Pediatrics had been working during the past year with what seemed to us to be less encouraging information concerning pediatric research. We were dealing with the fact that the NIH Institute with the major commitment to research concerning mothers and children. NICHD, had been able to fund only $36 \%$ of competing research grants during the past decade compared to $46 \%$ for NIH as a whole. We were dealing with the fact that the FY 1980 NIH budget would permit the funding of only $23 \%$ of competing grants within NICHD, compared to 37\% throughout NIH. The picture for FY 1981 looked no brighter. Moreover. FY 1981, now upon us, offered the projection of $25 \%$ fewer competing research grants than FY 1979 in NICHD, and not one new NICHD training grant, a critically important issue not touched on by Dr. Lowe.

Dr. Lowe's presentation in San Antonıo and his paper, now available in this issue of the journal for careful review, was and is an eloquent elucidation of the events at NIH during the past decade. Dr. Lowe makes a very succinct point that those investigators who direct their efforts toward the biomedical issues of mothers and children, pediatric investigators in the main, constitute approximately $3 \%$ of the total pool of researchers and attract an equivalent $3 \%$ of all the NIH funds for their investigative work tit for tat! These academic pediatric investigators, $3 \%$ of the academic whole throughout the United States, contribute another equivalent $3 \%$ of the pool of individuals participating in the peer review process at NIH. The symmetry of these data is remarkable and we are indebted to Dr. Lowe for retrieving comparative data in as plausible a fashion as might be possible. However, other data sources suggest that of the 35,000 faculty in the United States, 3100 are pediatricians, almost $10 \%$ of the whole rather than the $3 \%$ figure extrapolated by Dr. Lowe from the APSSPR membership. Dr. Lowe implies that all of the 35,000 full time faculty in the country are researchers: this is unlikely. Furthermore, the issue of pediatric participation in the peer review process, an issue of keen importance to APS-SPR-AMSPDC-AAP during the past year, may not be quite as favorable as depicted by Dr. Lowe in Figure 13 of his manuscript. Examining the comparative posture of our colleagues in internal medicine and equating the American Association of Physicians and the American Society of Clinical Investigation with the American Pediatric Society and the Society for Pediatric Research as research societies primarily for internists and pediatricians, respectively, with rigorous qualifications for membership, the mathematics shift substantially. The AAP and ASCI have 2694 members of whom 1405 serve on NIH Advisory Committees, or $52 \%$ rather than the $11 \%$ indicated in Figure 13, and to be contrasted with the $28 \%$ of APSSPR pediatricians serving in this capacity. The American Feder- ation for Clinical Research is a large multidisciplinary society without comparably rigorous scientific criteria for membership and should not be counted in Figure 13.

The majority of Dr. Lowe's statistics concerning research grants seem to match those available to many members of our academic, scientific, and societal pediatric community during the past year. Dr. Lowe's illustrative slides, used to highlight his remarks in San Antonio, are now available to the readership of the journal as figures and tables in his manuscript. They are complex and creative but much easier to analyze as manuscript figures than as slides. By and large. Dr. Lowe has transformed two-dimensional tabular data into near three-dimensional figures. I wish to dwell on some of these figures, wherein specific points of amplification and further translation can be made. Figure 8 depicts an impressive upsweep of the number of competing grants throughout NIH, including NCI, NHLBI, and NIAMDD, but not NICHD. Written between the curves in Figure 8 is the impact of special lobby groups such as cancer, heart, and diabetes, all of which are admittedly important for children. On the other hand, how effective has been the lobby for children themselves or mothers and children? Although Dr. Lowe states that the four NIH Institutes which generate the major funding for pediatric research. NCI. NHLBI, NIAMDD, and NICHD, "hover" around 45\% funding of competing grants, careful inspection of Figure 9 suggests that the Institute which contributes the major source of research dollars to pediatric departments. NICHD, has resided very clearly in the lower percentages of grant approval, from 36 to $38 \%$, during the last few years of the 1970s, whereas NIH as a whole and several of the other key Institutes attain percentages of grant approval which are almost $10 \%$ higher, consistent with the perception of the pediatric community prior to Dr. Lowe's compelling paper in San Antonio. Perhaps Dr. Lowe's most insightful piece of information is that illustrated by Figure 10, an almost stereoscopic illustration of important data, which indicates that departments of pediatrics, where presumably the majority of pediatric investigators are located, are securing rates of grant funding equivalent to that throughout the NIH process and other disciplines, approximately $45 \%$ during this past 5 -year span of the 1970s. So, most of the data coalesce most of the time. As many of us know, these data are difficult to extract, a web of numerators and denominators. some of the data subject to somewhat variable interpretation. Although we may conclude that $3 \%$ (or is it $10 \%$ ) of the national investigator pool, research-oriented pediatricians, garner $3 \%$ of the total research dollars and have $3 \%$ of the peer review voice, who else is focusing on research concerning children and their mothers? Is it the community of research obstetricians? Could it be other clinical scientists and basic scientists in all the preclinical fields? This is possibly so, but it seems unlikely to me. and I cannot find data to support or refute the point that another approximate $27 \%$ of the total scientific community has taken up the slack to produce scientific discovery of importance for children, the children who constitute approximately $30 \%$ of our populace. We might all conclude that this nation still falls considerably short of the mark. concerning dollars and investigators. necessary to address the needs of our children. In discussing pediatric research. Dr. Lowe 
made little or no comment about training grants. He also dwelt largely on the decade of the 70 s when our most substantial problems lay ahead of us in the $80 \mathrm{~s}$. There is reason to believe that we are slipping behind in research in general. There is reason to believe that pediatric research, certainly research concerning children and their mothers, is slipping even farther behind. There is also ample reason to suggest that we must renew our efforts to train a greater number of more skilled pediatric investigators. We cannot do that without competitive training grants, as well as research grants, in our most energetic and scientifically oriented pediatric departments. 